\title{
ON THE REAL PARTS OF THE ZEROS OF COMPLEX POLYNOMIALS AND APPLICATIONS TO CONTINUED FRACTION EXPANSIONS OF ANALYTIC FUNCTIONS
}

\author{
BY \\ EVELYN FRANK
}

1. Introduction. An arithmetic-algebraic process was given by Schur [7](1) to determine whether a polynomial with complex coefficients

$$
P(z) \equiv A_{0} z^{n}+A_{1} z^{n-1}+\cdots+A_{n}
$$

is a Hurwitz polynomial (2). This method is extended in this paper to an algorithm for counting the number of zeros of $P(z)$ with positive and negative real parts (\$2). A different method for the determination of these numbers has been given by Frank [3] and Bilharz [1] in terms of determinants and another by Frank [3] in terms of continued fractions.

In $\S 3$, a method is given for the computation of the zeros of $P(z)$ by an application of the algorithm of $\$ 2$ and the use of successive approximations.

A second application of the process of $\$ 2$ is found in an extension of the theory of Schur for continued fraction expansions of functions analytic in a half-plane and of certain rational functions ( $\$ 4)$.

In $\S 5$, a continued fraction expansion for functions analytic in $R(z)>0$ is obtained, and in $\$ 6$ partial fraction expansions are found for rational functions related to those discussed in $\$ \S 4$ and 5 .

2. The real parts of the zeros of a complex polynomial. Let $P(z)$ be the polynomial (1.1) of degree $n$ with complex coefficients, and

$$
P^{*}(z)=\bar{P}(-z),
$$

where $\bar{P}(z)$ is obtained by the replacement of the coefficients of $P(z)$ by their complex conjugates.

The following lemma is used in the proofs of subsequent theorems.

LEMma 2.1. If $\lambda$ is an arbitrary constant such that $|\lambda|<1$, then the two polynomials $P(z)$ and $P(z)+\lambda P^{*}(z)$ vanish the same number of times in $R(z)>0$ and in $R(z)<0\left(^{3}\right)$.

Presented to the Society, August 23,1946, and November 30,1946; received by the editors September 8, 1946.

(1) Numbers in brackets refer to the bibliography at the end of the paper.

(2) A polynomial whose zeros all have negative real parts.

(3) If $|\lambda|=1$, the zeros of $P(z)+\lambda P^{*}(z)$ are all on the imaginary axis, and if $|\lambda|>1$, the polynomials $P^{*}(z)$ and $P(z)+\lambda P^{*}(z)$ vanish the same number of times in each half-plane. Lemma 2.1 is an immediate consequence of a theorem of Rouche, Memoire sur la serrie de Lagrange, J. Ecole Polytech. vol. 22 (1862) pp. 193-224. 
Proof. For $|\lambda|<1$, the zeros of the polynomial

$$
P_{\lambda}(z)=P(z)+\lambda P^{*}(z)
$$

are continuous functions of $\lambda$. Consider the case $R(z)>0$. The number of zeros in $R(z)>0$ can change only if a zero crosses the imaginary axis. Then, for $z_{1}=i b$, since

$$
|P(i b)|=\left|P^{*}(i b)\right|,
$$

$P_{\lambda}\left(z_{1}\right)=P\left(z_{1}\right)+\lambda P^{*}\left(z_{1}\right)=0$, and consequently $\left|P\left(z_{1}\right)\right|<\left|P^{*}\left(z_{1}\right)\right|$, in contradiction to (2.2). Therefore the polynomials $P(z)$ and $P(z)+\lambda P^{*}(z)$ vanish the same number of times in $R(z)>0$. A similar proof holds for the number of zeros in $R(z)<0$.

It may be remarked that the polynomials $P(z)$ and $P(z)+\lambda P^{*}(z)$ also vanish the same nimber of times in each quadrant of the complex plane.

TheOREM 2.1. If $\xi$ is a complex constant such that

$$
\left|P^{*}(\xi)\right|>|P(\xi)| \text {, }
$$

then the polynomial of degree $n-1$,

$$
P_{1}(z) \equiv \frac{P^{*}(\xi) P(z)-P(\xi) P^{*}(z)}{z-\xi},
$$

has one zero less than $P(z)$ with real part of the same sign as $R(\xi)$ and the same number of zeros as $P(z)$ with real parts of opposite sign to $R(\xi)$.

Proof. By Lemma 2.1 and condition (2.3), the polynomial

$$
G(z) \equiv P^{*}(\xi) P(z)-P(\xi) P^{*}(z)
$$

has the same number of zeros as $P(z)$ in $R(z)>0$ and in $R(z)<0$. Also, $G(z)$ has a zero $z=\xi$. Consequently

$$
P_{1}(z)=\frac{G(z)}{z-\xi}
$$

is a polynomial with zeros distributed in $R(z)>0$ and in $R(z)<0$ as stated in the theorem.

As a special case of Theorem 2.1, Schur [7] proved the following.

THEOREM 2.2. If $R(\xi)<0$, the polynomial (1.1) is then and only then a Hurwitz polynomial if (2.3) holds and the polynomial (2.4) of degree $n-1$ is a Hurwitz polynomial.

If the modulus of the polynomial $P(z)$ is regarded as the product of a positive constant times the product of the lengths of the vectors from $z$ to its zeros, one concludes immediately that if $(2.3)$ holds for $\xi=a+i b$, then for 
$\xi_{1}=-a+i b$,

$$
\left|P^{*}\left(\xi_{1}\right)\right|<\left|P\left(\xi_{1}\right)\right| \text {. }
$$

For $z=i b,(2.2)$ holds. Therefore, any value $\xi$, not a zero of $P(z)$ or $P^{*}(z)$, for which (2.3) holds may be used to obtain $P_{1}(z)$.

If the polynomial $P(z)$ does not have pure imaginary zeros or pairs of zeros of the form $\pm \alpha_{j}+i \beta_{j}$, by a repetition of the process of Theorem 2.1 on the polynomial $P_{1}(z)$, and this again on the resulting polynomial $P_{2}(z)$ of degree $n-2$, after $n$ steps, $P_{n}(z)=$ constant is obtained. Then the number of zeros of the original polynomial $P(z)$ with positive real parts and the number with negative real parts are equal to the numbers of constants $\xi_{i}$ with positive and negative real parts, respectively.

If $P(z)$ has $p$ zeros which are either pure imaginary or are in pairs of the form $\pm \alpha_{j}+i \beta_{j}$, or both, then the above recurrent process leads, after $n-p$ steps, to a polynomial $P_{n-p}(z)$ with zeros $i \gamma_{k}$ and/or $\pm \alpha_{j}+i \beta_{j}$ since the polynomials $P(z), P_{1}(z), \cdots, P_{n-p}(z)$ all have these zeros. This step is reached if for every $\xi$,

$$
\left|P^{*}(\xi)\right|=|P(\xi)| .
$$

In this case, by the substitution $z^{\prime}=z+\epsilon$, where $\epsilon$ is an arbitrarily small positive real number, the number of zeros on the imaginary axis are easily distinguished from the pairs of zeros $\pm \alpha_{j}+i \beta_{j}$. Here these pairs remain separated by the imaginary axis, while the pure imaginary zercs are in $R\left(z^{\prime}\right)>0$.

These results may be summarized in the following rule.

RULE. The number of zeros of $P(z)$ with positive real parts and the number with negative real parts are equal to the numbers of constants $\xi_{i}$ with positive and negative real parts, respectively, which are used by a repetition of the process of Theorem 2.1. If some polynomial $P_{n-p}(z)$ is reached in the recurrent process such that (2.6) holds for every $\xi$, then after the substitution $z^{\prime}=z+\epsilon$, where $\epsilon$ is an arbitrarily small positive real number, the process may be carried out on $P_{n-p}\left(z^{\prime}\right)$ in order to find the total number of positive and negative real parts of $P(z)$.

3 . The computation of the zeros of a complex polynomial. The method of $\$ 2$ will now be applied to the actual computation of the zeros of $P(z)$.

By Horner's method the polynomials $P(z-c)$ are formed and by the algorithm of $\$ 2$ the number of zeros of $P(z)$ on either side of the line $R(z)=c$ are counted. If $c$ is varied successively, a zero of $P(z-c)$ can be made to fall on the imaginary axis by the method of $\$ 2$. The best values $c$ to be used at each step of the computation may be found by interpolation between those for which there is a change in the number of zeros on each side of the imaginary axis of $z^{\prime}=z-c$. When the best value of $c$ is found (if the value of $c$ is not exact, as in the example below) and the corresponding value of $d$ is computed as the zero of $P_{n-1}$, the zero of $P(z)$ is approximately $c+i d$. One may obtain a better approximation by forming $P[z-(c+i d)]$, and equating to zero the 
linear term of this polynomial. This correction, as in Horner's method, may be added to the value of $c+i d$ to give a better approximation to the zero. A still better approximation may be found by use of the second degree term of $P[z-(c+i d)]$.

The computation may be arranged as shown in the following example.

Let $P(z)=2 z^{3}+(1+i) z^{2}+(1+i) z+(-1+i)$. By the rule of $\$ 2$ it is found that $P(z)$ has two zeros with negative real parts and one with positive real part. To compute this latter zero, the polynomials $P(z-c)$ and the corresponding polynomials $P_{1}(z-c)$ and $P_{2}(z-c)$ are formed, and $c$ varied until at the last step $P(z-c)$ has two zeros to the left of the line $R(z)=c$ and one zero on $R(z)=c$. For this value of $c=.5$, we find the zero of $P_{2}(z-.5)$, namely $d=-.5$, as follows:

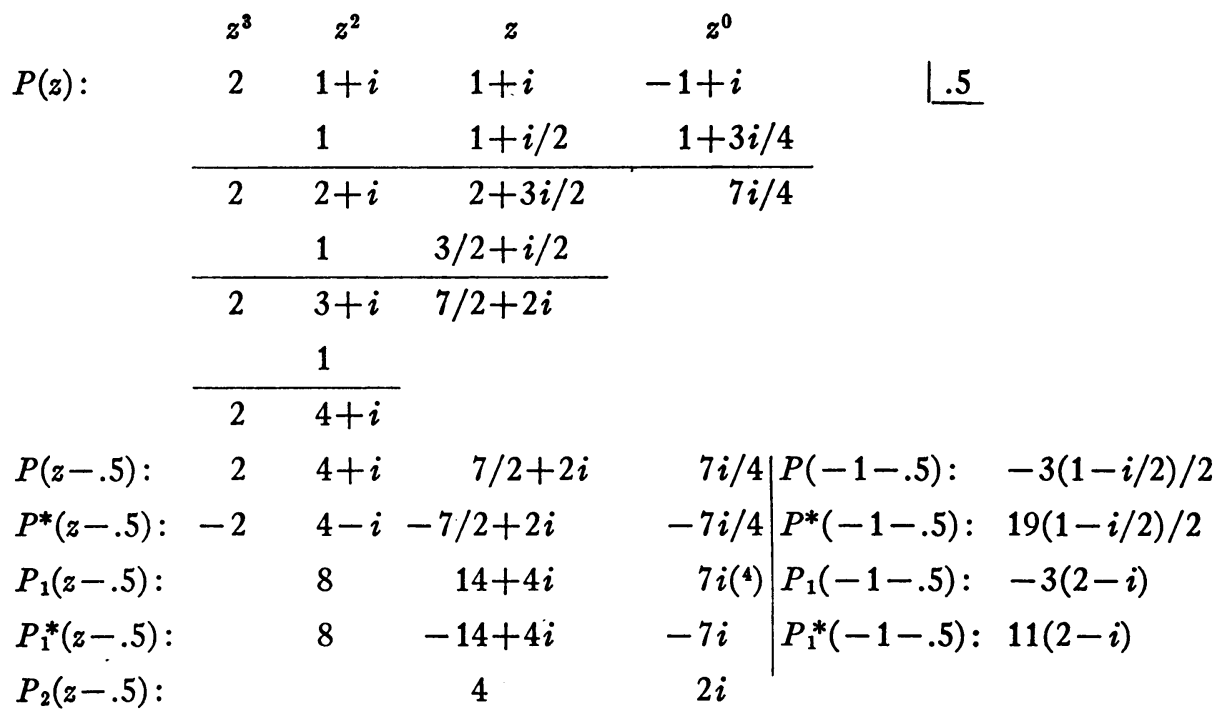

The zero of $P(z)$ with positive real part is therefore equal to $.5-.5 i$.

4. Functions analytic in a half-plane. The class $A$ of functions $f(z)$ which are analytic and have moduli less than or equal to one for $R(z)>0$ is first considered.

By the transformation $w=\sigma(z+\xi) /(z-\xi), R(\xi)<0, \sigma=1$, which maps the interior of the unit circle on $R(z)>0$, the theory of Schur [6] (cf. also Wall [8] and Geronimus [5]) may be carried over directly to functions analytic in $R(z)>0$ (or by other similar transformations to any half-plane). Under this transformation, then, to each function $f(z)$ there is related a finite or infinite sequence of Schur-type functions $f(z), f_{1}(z), f_{2}(z), \cdots$, also of the class $A$, between which there exist the recurrence relations

( $\left.{ }^{4}\right)$ To simplify the computation, all common constant factors of $P_{i}(z)$ and $P_{i}^{*}(z)$ may be dropped. 


$$
\begin{aligned}
f_{k+1}=\frac{(z-\xi)\left(\gamma_{k}-f_{k}\right)}{\sigma(z+\bar{\xi})\left(1-\bar{\gamma}_{k} f_{k}\right)}, \quad f_{k}=\frac{\gamma_{k}(z-\xi)-\sigma f_{k+1}(z+\bar{\xi})}{(z-\xi)-\sigma \bar{\gamma}_{k} f_{k+1}(z+\bar{\xi})}, \\
\gamma_{k}=f_{k}(-\bar{\xi}), \quad k=0,1,2, \ldots .
\end{aligned}
$$

If the sequence is infinite, the parameters $\gamma_{k}$ are such that $\left|\gamma_{k}\right|<1, k=0,1$, $2, \cdots$; if the sequence is finite, $\left|\gamma_{k}\right|<1, k=0,1, \cdots, n-1,\left|\gamma_{n}\right|=1$. In the linear transformations

$$
s=s_{k}(z, t)=\frac{\gamma_{k}(z-\xi)-\sigma(z+\bar{\xi}) t}{(z-\xi)-\sigma \bar{\gamma}_{k}(z+\bar{\xi}) t}
$$

of the $t$-plane into the $s$-plane, if $R(z)>0$, then $|t| \leqq 1$ implies $|s| \leqq 1$. Also for the product $S_{k}(z, t)=s_{0} s_{1} \cdots s_{k}(z, t)$,

$$
\left|S_{k}(z, t)\right| \leqq 1
$$

for $R(z)>0$ and $|t| \leqq 1$. The product $S_{k}(z, t)$ may be written in the form

$$
\begin{aligned}
\gamma_{0}+\frac{(z+\bar{\xi}) \sigma\left(1-\left|\gamma_{0}\right|^{2}\right)}{(z+\bar{\xi}) \sigma \bar{\gamma}_{0}} & -\frac{z-\xi}{\gamma_{1}}+\frac{(z+\bar{\xi}) \sigma\left(1-\left|\gamma_{1}\right|^{2}\right)}{(z+\bar{\xi}) \sigma \bar{\gamma}_{1}}-\cdots \\
& -\frac{z-\xi}{\gamma_{k}}+\frac{(z+\bar{\xi}) \sigma\left(1-\left|\gamma_{k}\right|^{2}\right)}{(z+\bar{\xi}) \bar{\gamma}_{k}}-\frac{z-\xi}{t} .
\end{aligned}
$$

For the infinite sequence the function $f(z)$ in $A$ is defined for $z$ in $R(z)>0$ by

$$
f(z)=\lim _{z \rightarrow \infty} S_{k}\left(z, t_{k}\right),
$$

and the limit exists uniformly over every bounded closed domain in $R(z)>0$. The $t_{k}$ are any sequence of functions with moduli not greater than 1 (Wall [8]). For the finite sequence, the function $f(z)$ is defined by $S_{n}\left(z, \gamma_{n}\right)$. Schur proved that this case occurs if and only if $f(z)$ is a rational function of a particular form (cf. [6, p. 208]). We shall now prove the analogous theorem where $f(z)$ is a function of the class $A$ by use of Theorem 2.2.

THEOREM 4.1. There is an integer $n$ for which

$$
\left|\gamma_{0}\right|<1,\left|\gamma_{1}\right|<1, \cdots,\left|\gamma_{n-1}\right|<1,\left|\gamma_{n}\right|=1
$$

and the sequence of functions $f_{k+1}(4.1)$ is finite if and only if $f(z)$ is a rational function of the form

$$
f(z)=\epsilon \frac{P(z)}{P^{*}(z)}, \quad|\epsilon|=1, P^{*}(z)=\bar{P}(-z),
$$

where $P^{*}(z)$ is a polynomial of degree $n$, vanishes only in $R(z)<0$, and for some constant $\xi$, where $R(\xi)<0, P^{*}(-\bar{\xi})=a$, a positive real constant, and $\sigma=1$. The 
function $f(z)$ may abso be expressed in the form

$$
\epsilon \prod_{v=1}^{n} \frac{-z+\bar{\beta}_{v}}{z+\beta_{v}}
$$

where $R\left(-\beta_{v}\right)<0$ and $\prod_{0-1}^{n}\left(-\bar{\xi}+\beta_{v}\right)=a$.

Proof. If $f(z)$ is of the form (4.6), it belongs to the class $A$ since $P^{*}(z) \neq 0$ for $R(z)>0$. To show that it can be expressed as a finite continued fraction of the form (4.3), where the $\gamma_{k}$ satisfy conditions (4.5), we note first that it may be thus expressed for $n=0$, since then $|f(z)|=|\epsilon|=1$. If, however, $n>0$, let

$$
f_{1}(z)=\epsilon \frac{P_{1}(z)}{P_{1}^{*}(z)}=\frac{P_{0}^{*}(-\bar{\xi})}{\bar{P}_{0}^{*}(-\xi)} \cdot \frac{(z-\xi)\left(\gamma_{0}-f_{0}\right)}{(z+\xi)\left(1-\bar{\gamma}_{0} f_{0}\right)}
$$

where

$$
\gamma_{0}=f_{0}(-\bar{\xi}), \quad \bar{\gamma}_{0}=\bar{f}_{0}(-\xi) .
$$

Since $P_{0}^{*}(-\bar{\xi})=a$, where $P \equiv P_{0}$, then $P_{0}^{*}(-\bar{\xi})=\bar{P}_{0}^{*}(-\xi)=a$. Since $\left|P_{0}^{*}(-\xi)\right|$ $>\left|\bar{P}_{0}(-\xi)\right|$, we have $\left|\bar{\gamma}_{0}\right|<1$. Then by Theorem $2.2, P_{1}^{*}(z)$ is a polynomial of degree $n-1$ and cannot vanish in $R(z)>0$, and $P_{1}(z)$ is also a polynomial of degree $n-1$ which does not vanish in $R(z)<0$. The function $f_{1}(z)$ is therefore of the same form as $f(z)$ and belongs to the class $A$. By forming all the functions

$$
\begin{aligned}
f_{k+1}(z)=\epsilon \frac{P_{k+1}(z)}{P_{k+1}^{*}(z)}=\frac{P_{k}^{*}(-\bar{\xi})}{\bar{P}_{k}^{*}(-\xi)} \cdot \frac{(z-\xi)\left(\gamma_{k}-f_{k}\right)}{(z+\xi)\left(1-\bar{\gamma}_{k} f_{k}\right)}, \\
k=0,1, \cdots, n-1,
\end{aligned}
$$

where

$$
\gamma_{k}=f_{k}(-\bar{\xi}), \quad \bar{\gamma}_{k}=\bar{f}_{k}(-\xi),
$$

since $P_{0}^{*}(-\bar{\xi})=a, P_{k}^{*}(-\bar{\xi})=\bar{P}_{k}^{*}(-\xi)$, we obtain a finite sequence of functions (4.1), each of the class $A$, with $\left|f_{n}\right|=\left|\gamma_{n}\right|=|\epsilon|=1, \sigma=1$. Thus a terminating continued fraction expansion of the form (4.3) is obtained for $f(z)$.

If, conversely, $f(z)$ is a function of the class $A$ with parameters $\gamma_{k}$ which satisfy conditions (4.5), then $f_{n}(z)=\gamma_{n}$, where $\left|\gamma_{n}\right|=1$. It has been shown above that $f_{p+1}=\epsilon P_{p+1}(z) / P_{p+1}^{*}(z)$, where $P_{p+1}^{*}(z)$ is a polynomial of degree $n-p-1, P_{p+1}^{*}(-\bar{\xi})=\bar{P}_{p+1}^{*}(-\xi)$, and $P_{p+1}^{*}(z) \neq 0$ for $R(z)>0$. Then

$$
f_{p}=\frac{(z-\xi) \gamma_{p}-(z+\xi) f_{p+1}}{(z-\xi)-(z+\bar{\xi}) \bar{\gamma}_{p} f_{p+1}}=\epsilon \frac{P_{p}(z)}{P_{p}^{*}(z)}
$$

where 


$$
P_{p}^{*}(z)=(z-\xi) P_{p+1}^{*}(z)-\epsilon(z+\bar{\xi}) \bar{\gamma}_{p} P_{p+1}(z) .
$$

This polynomial is of degree $n-p$ and satisfies the condition $P_{p}^{*}(-\bar{\xi})$ $=\bar{P}_{p}^{*}(-\xi)$. By Theorem 2.2, $P_{p}^{*}(z) \neq 0$ for $R(z)>0$. Thus the properties which hold for $P_{p+1}^{*}(z)$ also hold for $P_{p}^{*}(z)$, and by a continuation of this argument they also hold for $P_{0}^{*}(z)$. Therefore $f(z)$ must be of the form (4.6).

We remark that a necessary and sufficient condition for a rational function $f(z)=B(z) / A(z)$, where $B(z)$ and $A(z)$ are polynomials of degree $n$ and $A(z)$ does not vanish in $R(z)>0$, to have a continued fraction expansion of the form (4.3) is that

$$
A(z) A^{*}(z)-B(z) B^{*}(z)=k,
$$

where $k$ is a non-negative real constant. The proof is entirely analogous to that found in [6] for the case $|z|<1$.

A finite continued fraction expansion for a larger class of rational functions than that found by Schur is obtained in the following theorem by the application of the algorithm of Theorem 2.1.

THEOREM 4.2. The constants $\gamma_{k}$ satisfy (4.5) and the sequence of functions

$$
\begin{aligned}
& f_{k+1}=\frac{\left(z-\xi_{k}\right)\left(\gamma_{k}-f_{k}\right)}{\sigma_{k}\left(z+\bar{\xi}_{k}\right)\left(1-\bar{\gamma}_{k} f_{k}\right)}, \\
& \quad \gamma_{k}=f_{k}\left(-\xi_{k}\right),\left|\sigma_{k}\right|=1, k=0,1, \cdots, n-1,
\end{aligned}
$$

is finite if and only if $f(z)$ is a rational function of the form (4.6), where $P^{*}(z)$ is a polynomial of degree $n$.

Proof. The proof follows in exactly the same way as that of Theorem 4.1, with the exception that the $\xi_{k}$ are chosen as any complex constants such that $P_{k}^{*}\left(\xi_{k}\right) \neq 0$ and $\left|\bar{P}_{k}^{*}\left(-\xi_{k}\right)\right|>\left|\bar{P}_{k}\left(-\xi_{k}\right)\right|$. Here $\bar{P}_{k}^{*}\left(-\xi_{k}\right) / P_{k}^{*}\left(-\bar{\xi}_{k}\right)=\sigma_{k}$. Consequently at each step of the recurrent process, according to Theorem 2.1 , a polynomial of one lower degree is obtained in the numerator and denominator of the rational function.

The finite continued fraction which is obtained in this case is of the form

$$
\begin{aligned}
\gamma_{0}+\frac{\left(z+\bar{\xi}_{0}\right) \sigma_{0}\left(1-\left|\gamma_{0}\right|^{2}\right)}{\left(z+\xi_{0}\right) \sigma_{0} \bar{\gamma}_{0}}-\frac{z-\xi_{0}}{\gamma_{1}}+\frac{\left(z+\bar{\xi}_{1}\right) \sigma_{1}\left(1-\left|\gamma_{1}\right|^{2}\right)}{\left(z+\bar{\xi}_{1}\right) \sigma_{1} \bar{\gamma}_{1}} \\
-\frac{z-\xi_{1}}{\gamma_{2}}+\cdots+\frac{\left(z+\bar{\xi}_{n-1}\right) \sigma_{n-1}\left(1-\left|\gamma_{n-1}\right|^{2}\right)}{\left(z+\bar{\xi}_{n-1}\right) \sigma_{n-1} \bar{\gamma}_{n-1}}-\frac{z-\xi_{n-1}}{\gamma_{n}} .
\end{aligned}
$$

'We remark that if $\xi$ in Theorem 4.1 is chosen so that $R(\xi)<0$ but $P^{*}(-\bar{\xi})$ $\neq a$, then the continued fraction expansion for the function (4.6) takes the form (4.10) with all $\xi_{k} \equiv \xi$.

On the basis of the results of Schur outlined here, we shall now obtain a continued fraction expansion for functions analytic in $R(z)>0$. This extends 
the expansions found in [8] for functions with only positive real parts.

5. A continued fraction expansion for functions analytic in $R(z)>0$. Let $B$ denote the class of all functions $F(z)\left({ }^{5}\right)$ which are analytic in $R(z)>0$ and are equal to unity for $z=-\bar{\xi}, R(\xi)<0$. If

$$
F(z)=\frac{\Delta_{0}(z-\xi)+(z+\bar{\xi}) f(z)}{\Delta_{0}(z-\xi)-(z+\xi) f(z)}, \quad\left|\Delta_{0}\right| \leqq 1, \Delta_{0} \neq \frac{(z+\bar{\xi}) f(z)}{(z-\xi)},
$$

there is a one-to-one correspondence between the functions $f(z)$ of the class $A$ and the functions $F(z)$ of the class $B$, since

$$
R(F(z))=\frac{\left|\Delta_{0}\right|^{2}-|(z+\bar{\xi}) f(z) /(z-\xi)|^{2}}{\left|\Delta_{0}-(z+\bar{\xi}) f(z) /(z-\xi)\right|^{2}} .
$$

Then $R(F(z))>0$ if $\left|\Delta_{0}\right|>|(z+\bar{\xi}) f(z) /(z-\xi)|, R(F(z))<0$ if $\left|\Delta_{0}\right|<\mid(z+\bar{\xi})$ $\cdot f(z) /(z-\xi) \mid$ and $R(F(z))=0$ if $\left|\Delta_{0}\right|=|(z+\bar{\xi}) f(z) /(z-\xi)|$.

A continued fraction expansion for functions of the class $B$ is obtained by a process similar to that outlined in [8] for functions analytic in a certain domain and with positive real parts. The functions $h_{k}(z, t)$ are defined by means of the relation

$$
h_{k}(z, t)=\frac{\Delta_{k}-f_{k}(z, t)}{\Delta_{k}+(z+\bar{\xi}) f_{k}(z, t) /(z-\xi)}, \quad k=0,1, \cdots, p,
$$

where the constants $\Delta_{k}$ are to be determined. By substitution of the values of $f_{k}$ frcm (4.1), then

$$
h_{k}=\frac{\Delta_{k}-\gamma_{k}}{\Delta_{k}-\Delta_{k+1}(z+\xi) /(z-\xi)+h_{k+1}\left(\Delta_{k+1}+\gamma_{k}\right)(z+\bar{\xi}) /(z-\xi)}
$$

if

$$
\Delta_{k+1}=\frac{\Delta_{k}-\gamma_{k}}{1-\Delta_{k} \bar{\gamma}_{k}}, \quad k=0,1, \cdots, p-1
$$

Since $f_{0}(z, t)=S_{p}(z, t)$,

$$
\begin{aligned}
h_{0} & =\frac{\Delta_{0}-S_{p}}{\Delta_{0}+(z+\xi) S_{p} /(z-\xi)} \\
& =\frac{\Delta_{0}-\gamma_{0}}{\Delta_{0}-\Delta_{1}(z+\bar{\xi}) /(z-\xi)+h_{1}\left(\Delta_{1}+\gamma_{0}\right)(z+\bar{\xi}) /(z-\xi)} .
\end{aligned}
$$

On multiplying both sides of this equation by $2(z+\bar{\xi}) /(-\xi-\bar{\xi})$, adding 1 to

(5) The functions $F(z)$ are generalizations of the Carath eodory functions [1] (transformed from the unit circle to the right half-plane) which are regular in and on the unit circle, have positive real parts for these values, and have $F(0)=1$. 
both sides, and taking reciprocals, one obtains

$$
\begin{aligned}
& \frac{\Delta_{0}+}{\Delta_{0}-}(z, t)(z+\bar{\xi}) /(z-\xi) \\
& \quad=\frac{z+(z, t)(z+\xi) /(z-\xi) / 2}{-(\xi+\xi) / 2}+\frac{(z+\xi)(z-\xi)\left(\Delta_{0}-\gamma_{0}\right)}{\Delta_{0}(z-\xi)-\Delta_{1}(z+\xi)+h_{1}(z+\xi)\left(\Delta_{1}+\gamma_{0}\right)} .
\end{aligned}
$$

Since $f_{p}(z, t)=t$,

$$
h_{p}=\frac{\Delta_{p}-t}{\Delta_{p}+t(z+\xi) /(z-\xi)} .
$$

If the $\gamma_{p}$ form an infinite sequence, for $t=t_{p}=\Delta_{p}, h_{p} \equiv 0$. Then by (5.3)

$$
\frac{\Delta_{0}+S_{p}\left(z, t_{p}\right)(z+\xi) /(z-\xi)}{\Delta_{0}-S_{p}\left(z, t_{p}\right)(z+\xi) /(z-\xi)}
$$

is the $(p+1)$ th approximant of the continued fraction expansion

$$
\begin{aligned}
\frac{z+(\xi-\xi) / 2}{-(\xi+\xi) / 2}+ & \frac{(z+\xi)(z-\xi)\left(\Delta_{0}-\gamma_{0}\right)}{\Delta_{0}(z-\xi)-\Delta_{1}(z+\xi)} \\
+ & \frac{(z+\xi)(z-\xi)\left(\Delta_{1}+\gamma_{0}\right)\left(\Delta_{1}-\gamma_{1}\right)}{\Delta_{1}(z-\xi)-\Delta_{2}(z+\bar{\xi})}+\cdots
\end{aligned}
$$

and, by (4.2) and (4.4),

$$
\lim _{p \rightarrow \infty} \frac{\Delta_{0}+S_{p}\left(z, t_{p}\right)(z+\xi) /(z-\xi)}{\Delta_{0}-S_{p}\left(z, t_{p}\right)(z+\xi) /(z-\xi)}=\frac{\Delta_{0}+f_{0}(z+\bar{\xi}) /(z-\xi)}{\Delta_{0}-f_{0}(z+\bar{\xi}) /(z-\xi)}=F(z)
$$

uniformly over any bounded closed domain in $R(z)>0$. If the $\gamma_{p}$ form a finite sequence, then

$$
F(z)=\frac{\Delta_{0}+S_{n}\left(z, \gamma_{n}\right)(z+\xi) /(z-\xi)}{\Delta_{0}-S_{n}\left(z, \gamma_{n}\right)(z+\xi) /(z-\xi)}
$$

a function of the class $B$, is equal to a finite continued fraction expansion of the form (5.4).

Conversely, for every function $F(z)$ there is determined uniquely an infinite or terminating continued fraction (5.4). Consequently, the following theorem holds.

THEOREM 5.1. The finite or infinite continued fraction (5.4) converges uniformly over every bounded closed domain in $R(z)>0$ to a function which is analytic in $R(z)>0$. Conversely, any function which is analytic for $R(z)>0$ is the value of a uniquely determined infinite or terminating continued fraction of the form (5.4). 
For the special case $\Delta_{0}=1,\left|\Delta_{k}\right|=1, k=1,2, \cdots$, Theorem 5.1 reduces after some transformations to the theorem in [8].

In order to obtain expansions of the form (5.4) for $F(z)$, it is not necessary to choose $\xi$ so that $F(-\bar{\xi})=1$, but merely so that $R(\xi)<0$.

6. Partial fraction expansions for certain rational functions. The functions $\phi_{1}(z)=\left[1-f_{1}(z)\right] /\left[1+f_{1}(z)\right]$ for

$$
f_{1}(z)=\epsilon \prod_{v=1}^{n} \frac{z+\omega_{v}}{1+\bar{\omega}_{v} z}=\epsilon \frac{P(z)}{Q(z)}, \quad\left|\omega_{v}\right|<1,|\epsilon|=1,
$$

are rational functions which have a partial fraction expansion of the form

$$
\phi_{1}(z)=b i-\sum_{v=1}^{n} r_{v} \frac{z+\epsilon_{v}}{z-\epsilon_{v}}
$$

where $b$ is real, the $\epsilon_{v}$ are the zeros of $\epsilon P(z)+Q(z)$ and are different from each other, $\left|\epsilon_{v}\right|=1$, and the $r_{v}$ are positive real numbers (cf. Schur [6]). We shall now find the particular form which the analogous partial fraction expansion takes for the functions

$$
\phi(z)=\frac{\lambda-f(z)}{\lambda+f(z)},
$$

where $\lambda$ is a complex constant, and

$$
f(z)=\epsilon \frac{P(z)}{P^{*}(z)}=\epsilon \prod_{v=1}^{n} \frac{-z+\bar{\beta}_{v}}{z+\beta_{v}}, \quad|\epsilon|=1, P^{*}(z)=\bar{P}(-z),
$$

where $P^{*}(z)$ is a polynomial of degree $n$.

THEOREM 6.1. The zeros $\epsilon_{v}$ of

$$
\psi(z)=\lambda P^{*}+\epsilon P
$$

are different provided

$$
\sum_{p=1}^{n} \frac{\bar{\beta}_{p}+\beta_{p}}{-\epsilon_{v}+\epsilon_{v}\left(\bar{\beta}_{p}-\beta_{p}\right)+\bar{\beta}_{p} \beta_{p}} \neq 0
$$

Then, if

(6.4) (i) $\lambda \neq \epsilon$, and $n$ is odd, or (ii) $\lambda \neq-\epsilon$, and $n$ is even, the rational functions (6.1) are expressible in a partial fraction expansion

$$
\phi(z)=c-\sum_{v=1}^{n} r_{v} \frac{z+\epsilon_{v}}{z-\epsilon_{v}},
$$

where for $|\lambda|=1$ the constants $c$ and the $r_{v}$ are pure imaginary, while for $|\lambda| \neq 1$ these values are in general of the form $a+i b$, where $a, b \neq 0$. If (6.4) (i) or (ii) 
does not hold, the functions (6.1) may be written in the form

$$
\phi(z)=Q(z)+c-\sum_{v=1}^{n-k} r_{v} \frac{z+\epsilon_{v}}{z-\epsilon_{v}}
$$

where the constants $c$ and the $r_{v}$ are pure imaginary and $Q(z)$ is a polynomial of degree $k$ with coefficients of the terms of even degree pure imaginary and coeffcients of the terms of odd degree real numbers.

Proof. Let us consider first the case where conditions (6.4) hold and $|\lambda|=1$. We may write $\phi(z)=\left[\lambda P^{*}-\epsilon P\right] /\left[\lambda P^{*}+\epsilon P\right]$. By Lemma 2.1 , the zeros $\epsilon_{v}$ of $\psi(z)$ all lie on the imaginary axis. Since $\lambda P^{*}\left(\epsilon_{v}\right)+\epsilon P\left(\epsilon_{v}\right)=0$,

$$
\begin{aligned}
\frac{\psi^{\prime}\left(\epsilon_{v}\right)}{\epsilon P\left(\epsilon_{v}\right)} & =\frac{-\lambda P^{* \prime}\left(\epsilon_{v}\right)}{\lambda P^{*}\left(\epsilon_{v}\right)}+\frac{\epsilon P^{\prime}\left(\epsilon_{v}\right)}{\epsilon P\left(\epsilon_{v}\right)}=\sum_{p=1}^{n}\left(-\frac{1}{\epsilon_{v}+\beta_{p}}+\frac{1}{\epsilon_{v}-\bar{\beta}_{p}}\right) \\
& =\sum_{p=1}^{n}-\frac{\bar{\beta}_{p}+\beta_{p}}{-\epsilon_{v}^{2}+\epsilon_{v}\left(\bar{\beta}_{p}-\beta_{p}\right)+\bar{\beta}_{p} \beta_{p}} .
\end{aligned}
$$

If $R\left(\beta_{p}\right)>0$, this number is real and negative. Then $\psi^{\prime}\left(\epsilon_{v}\right)$ is not zero, and the $\epsilon_{v}$ are all different from each other. Since we may write

$$
\frac{\lambda P^{*}-\epsilon P}{\lambda P^{*}+\epsilon P}=c-\sum_{v=1}^{n} r_{v} \frac{z+\epsilon_{v}}{z-\epsilon_{v}}
$$

then

$$
-2 \epsilon_{v} r_{v}=\frac{\lambda P^{*}\left(\epsilon_{v}\right)-\epsilon P\left(\epsilon_{v}\right)}{\lambda P^{* \prime}\left(\epsilon_{v}\right)+\epsilon P^{\prime}\left(\epsilon_{v}\right)}=\frac{-2 \epsilon P\left(\epsilon_{v}\right)}{\psi^{\prime}\left(\epsilon_{v}\right)}
$$

and the $r_{v}$ are pure imaginary numbers. If in (6.8) we substitute $z=i \delta$ (different from $\epsilon_{v}$ ), where $\delta$ is real, the expression on the left and every term on the right become pure imaginary. Consequently $c$ is pure imaginary. From (6.7) we see that the restriction $R\left(\beta_{p}\right)>0$ may be replaced by condition (6.3).

If $|\lambda| \neq 1$, the $\epsilon_{v}$ aie in general complex numbers. If (6.3) holds, the above argument shows that $c$ and the $r_{v}$ are in general also of the form $a+i b$, where $a$, $b \neq 0$.

If condition (6.4) (i) or (ii) does not hold, we may write

$$
\frac{\lambda P^{*}-\epsilon P}{\lambda P^{*}+\epsilon P}=Q(z)+c-\sum_{v=1}^{n-k} r_{v} \frac{z+\epsilon_{v}}{z-\epsilon_{v}},
$$

where $Q(z)$ is the quotient obtained by long division in the left-hand side of (6.9) until a remainder of the same degree as the divisor is obtained, and is a polynomial of degree $k$ with coefficients of even powers of $z$ pure imaginary and coefficients of odd powers real. By the same argument as above for $|\lambda|=1$, we see that $c$ and the $r_{v}$ are pure imaginary. 
A continued fraction expansion for the function $\phi(z)$ may be written in the form

$$
\phi(z)=1-\frac{2}{1}+\frac{\lambda}{f(z)}
$$

where $f(z)$ is the continued fraction (4.10).

We now consider the functions of $\$ 5$,

$$
F(z)=\frac{\Delta_{0}(z-\xi)+(z+\xi) f(z)}{\Delta_{0}(z-\xi)-(z+\xi) f(z)}, \quad\left|\Delta_{0}\right| \leqq 1,
$$

where $f(z)$ is given by (6.2). If we write

$$
\chi(z)=-\frac{\epsilon P_{1}(z)}{P_{1}^{*}(z)}=-\frac{z+\bar{\xi}}{z-\xi} f(z),
$$

then

$$
F(z)=\frac{\Delta_{0}-\chi(z)}{\Delta_{0}+\chi(z)}
$$

which is of the form (6.1). Consequently Theorem 6.1 applies to the functions $F(z)$ with $\lambda$ replaced by $\Delta_{0}, f(z)$ by $\chi(z)$, and $n$ by $n+1$.

\section{BIBLIOGRAPHY}

1. H. Bilharz, Bemerkung zu einem Satze von Hurwitz, Zeitschrift für angewandte Mathematik und Mechanik vol. 24 (1944) pp. 77-82 (lithoprinted by Edwards Brothers, Ann Arbor, Mich., 1945).

2. C. Carathéodory, Über den Variabilitatsbereich der Koeffizienten von Potensreihen, die gegebene Werte nicht annehmen, Math. Ann. vol. 64 (1907) pp. 95-115.

3. E. Frank, On the zeros of polynomials with complex coefficients, Bull. Amer. Math. Soc. vol. 52 (1946) pp. 144-157.

4. - The location of the zeros of polynomials with complex coefficients, Bull. Amer. Math. Soc. vol. 52 (1946) pp. 890-898.

5. J. Geronimus, On polynomials orthogonal on the circle, on trigonometric moment-problem and on allied Caratheodory and Schur functions, preliminary announcement, C. R. (Doklady) Acad. Sci. URSS. vol. 39 (1943) pp. 291-295; Rec. Math. (Mat. Sbornik) N.S. vol. 15 (1944) pp. 99-130.

6. I. Schur, Über Potenzreihen, die im Innern des Einheitskreises beschrankt sind, J. Reine Angew. Math. vol. 147 (1917) pp. 205-232; vol. 148 (1918) pp. 122-145.

7. - Über algebraische Gleichungen, die nur Wurzeln mit negativen Realteilen besitzen, Zeitschrift für angewandte Mathematik und Mechanik vol. 1 (1921) pp. 307-311.

8. H. S. Wall, Continued fraction expansions for functions with positive real parts, Bull. Amer. Math. Soc. vol. 52 (1946) pp. 138-143.

UNIVERSITY OF IlLINOIS,

Chicago, Ill. 